\title{
TOAST Classification
}

National Cancer Institute

\section{Source}

National Cancer Institute. TOAST Classification. NCI Thesaurus. Code C49121.

A classification of subtypes of ischemic stroke based on clinical features and the results of ancillary diagnostic studies.T he TOAST classification denotes five subtypes of ischemic stroke: 1) large-artery atherosclerosis, 2) cardioembolism, 3) small-vessel occlusion, 4) stroke of other determined etiology, and 5) stroke of undetermined etiology, 